\title{
Quantum electro-dynamics helps homoeopathy achieve its scientific basis
}

\begin{abstract}
Since long liberal minded scientists are trying to unravel the mystery of high potency (greater than 12c) homoeopathic medicines - how do they differ from one another and how do they cure diseases. Many investigations have been done by reputed scientists using sophisticated instruments. Now these attempts seem to converge to the structural model based on a few striking properties of water observed experimentally. The model may be stated as: A substance is to be recognized as a medicine if it has the capability of curing disease(s) while its medicinal property is to be attributed to molecular structure of vehicle like water or of distinct chemical substance when it exists. Aim of this article is to show that Quantum Electro-Dynamics (QED) gives the required theoretical support for those properties of water and overcomes the theoretical hurdle also towards scientific basis of homoeopathy.
\end{abstract}

Keywords: structural model, theoretical support, scientific basis of homoeopathy
Volume 3 Issue 6 - 2016

\author{
Mahata CR \\ Hony Emeritus Scientist, IIEST Shibpur, India
}

Correspondence: CR Mahata, Hony Emeritus Scientist, IIEST Shibpur, India, Tel +9l 943373 9180, Email crmahata@gmail.com

Received: June 30, 2016 | Published: July 14, 2016

\section{Introduction}

High potency (greater than12c) homoeopathic medicines are necessarily devoid of the starting medicinal substances as Avogadro number suggests. Chemically they cannot be anything except the diluting substance, that is, water. Still, we have the evidence that different such potentised substances act differently on living bodies. How the chemical-free water was accomplishing this job appeared to be incredible and mysterious for over two centuries. How to explain it was a great hurdle before the scientists. Thanks to the works of several scientists solution of the mystery seems to be in sight now.

\section{Quantum electro-dynamics homoeopathy}

and

It is obvious that water must have some special property by which it can hold some kind of medicine-plus-potency specific impression/ information in its molecular structures. Such an obvious thing was not being given due importance. Based on three special properties of water, observed experimentally, Mahata CR proposed a structural model. ${ }^{1,2}$ Experimental supports to this model are available in papers. ${ }^{2-8}$ But, now Quantum Electro Dynamic (QED) investigation of water lays the foundation of the properties on which Mahata based the structural model. Scientists like Guidance ED and his co-workers ${ }^{9-15}$ established a two-state model of water in which a substantial fraction of the molecules exist in hydrogen bonded state as coherent domains (CD) resembling that of ordinary ice. Thus the earlier finding of 'icicles' 16 in ordinary water gets QED-support. In a number of publications Konovalov AI and his group have shown presence of nanoassociates, that is, CD's ${ }^{17-24}$ in diluted aqueous solutions.

How do the nanoassociates or CD's present in water further help homoeopathy achieve its scientific basis? Forms of these crystals are so varied in number that practically no two ice-crystals are identical. ${ }^{25,26}$ It is found that each natural snow flake is distinct. ${ }^{26}$ Now, these icicles or ice-crystals can be identified as CD's with our knowledge augmented by QED. Further, the shape and size of these molecular clusters are influenced by impurities, ions of other substances and even foreign molecules. ${ }^{16,25}$ And these influencing objects can very well be homoeopathic medicines in low or high potencies. A decisive amount of experimental data has also been collected concerning the physicochemical properties of water subjected to physical perturbations like Iteratively Nafionated Water $(I N W),{ }^{27-29}$ Iteratively Filtered Water (IFW), ${ }^{29-32}$ effect of hydrophilic surfaces $^{33,34}$ and Extremely Diluted Solutions (EDS) ${ }^{35-40}$ Roy Rustum, Rey L, Elia $\mathrm{V}^{41-43}$ also indicate induced changes in structure of water. These are direct or indirect contributions in proving the existence of Coherent Domains (CD) in water. Prospect of water holding the key for unfolding the mystery has significantly brightened. Existence of $\mathrm{CD}$ means existence of stable water-clusters variously speculated as water polymers ${ }^{44}$ water clathrates ${ }^{45}$ and induced water structures. ${ }^{1,2}$ These water-clusters are shown to be specific to homoeo- medicines and their potencies. ${ }^{2,3,5-8}$

High dilution homoeopathic medicines continue to cure many diseases since the time of its discoverer S.C.F. Hahnemann. And if such medicines are nothing but structured water, then the obvious inference is that water structures do serve as medicines. However critics may demand a more direct proof - Such proof is available for antibiotics. ${ }^{46,47}$ Investigations are required to establish this point for homoeopathy. But quite a few indirect proofs are available to us. If chemistry was the sole deciding factor, then we could not have Carbo-animalis and Graphites as two separate medicines in homoeopathy since the starting materials for both are chemically nothing but carbon. They differ in the structural arrangement of their constituent atoms. The homoeopathic medicine called X-ray does not have any chemistry even in its lowest potency. Structural change of molecular arrangement of the vehicle stands out as the only possible explanation. Homoeopathic medicines exposed to direct sunlight lose their medicinal value. Its only explanation left to us is melting/ disintegration of structures of the vehicle or the preservative at high temperature. It is likely to be similar to loss of structures of doped semiconductors getting melted at high temperature. Homoeopathic medicines simply diluted in water (that is, without any preservative like ethyl alcohol) lose their medicinal value after 3-4 days. This may be explained by spontaneous disintegration and reorganization of water structures at room temperature. 


\section{Acknowledgements}

None.

\section{Conflict of interest}

The author declares no conflict of interest.

\section{References}

1. Mahata CR. Homoeopathy explained in the light of recognised science. The Homoeopathic Heritage. 1997;22:245-252

2. Maity Tanmoy, Ghosh D, Mahata CR. Theory and Instrumentation related to Potentised Homoeopathic Medicines. IE(I). 2007;88:27-31.

3. Maity Tanmoy, Ghosh D, Mahata CR. Theory and Instrumentation related to anomalous dielectric dispersion in ordered molecular groups. Sensors and Transducers. 2007;58(11):1745-1756.

4. Maity T, Ghosh D, Mahata CR. In Search Of A Technique For Identifying Potentised Homoeo-Medicines Beyond Avogadro Limit. Indian Journal of Research in Homoeopathy. 2010;4(3):1-9.

5. T Maity, D Ghosh, Mahata CR. Effect of Dielectric Dispersion on Potentised Homoeo-Medicines. Homeopathy. 2010;99(2):99-103.

6. Mahata CR. Dielectric Dispersion Studies Indicate Change in Structure of Water by Potentised Homeopathic Medicines. IE(I). 2013;93(4):231235 .

7. Mahata CR. Dielectric Dispersion Studies of Some Potentised Homoeopathic Medicines Reveal Structured Vehicle. Homeopathy. 2013;102(4):262-267.

8. Mahata CR. Structural model explains medicinal value of high potencies and leads to a unified understanding of medicines. Int J Complement Alt Med. 2015;1(2):1-3.

9. Marchettini N, Giudice E Del, Voeikov V, et al. Water: A medium where dissipative structures are produced by a coherent dynamics. J Theor Biol. 2010;265(4):511-516.

10. Giudice ED, Preparata G, Vitiello G. Water as a free electric dipole laser. Phys Rev Lett. 1988;61(9):1085-1088.

11. Arani R, Bono I, Giudice ED, et al. QED Coherence and the thermodynamics of water. Int J Modern Phys. 1995;139:1813-1841.

12. Bano I, Giudice ED, Gamberale L, et al. Emergence of the Coherent Structure of Liquid Water. Water. 2012;4:510-532.

13. Capolupo A, Giudice ED, Elia V, et al. Self-similarity properties of nafionized and filtered water and deformed coherent states. Int J Mod Phys B. 2014:28(3).

14. Giudice ED, Tedeschi A, Vitiello G, et al. Coherent structures in liquid water close to hydrophilic surfaces. J Phys Conf Ser. 2013;442:1-6.

15. Montagnier L, Giudice ED, Jamal A, et al. Transduction of DNA information through water and electromagnetic waves. Electromagnetic Biology and Medicine. 2014;34(2):1-11.

16. Sergeev B. Physiology for Everyone. Russia: Mir Publishers; 1971. p. $11-16$.

17. Ryzhkina IS, Kiseleva Yu V, Murtazina LI, et al. Comparative study of self-organization and physicochemical properties of highly diluted aqueous solutions of phenol bioantioxidants. Doklady Akademii Nauk. 2012;447(2):179-182.

18. Konovalov AI. The formation of nanosized molecular ensembles in highly dilute aqueous solutions. The Rostrum of the RAS Presidium. 2013;83(12):1076-1082.

19. Konovalov AI, Ryzhkina IS. Formation of nanoassociates as a key to understanding of physicochemical and biological properties of highly dilute aqueous solutions. Russian chem Bulletin. 2014;63(1):1-14.
20. Konovalov AI, Ryzhkina IS, Murtazina LI, et al. Forming the nanosized molecular assemblies (nanoassociates) is a key to understand the properties of highly diluted aqueous solutions. Biophysics. 2014;59(3):341-346.

21. Konovalov AI, Mal'tseva EL, Ryzhkina IS, et al. Formation of Nanoassociates Is a Factor Determining Physicochemical and Biological Properties of Highly Diluted Aqueous Solutions. Doklady Physical Chemistry. 2014;456(2):561-564.

22. Ryzhkina IS, Kiseleva Yu V, Mishina OA, et al. Highly diluted solutions of amphiphilic derivatives of calyx [4]resorcinols:Self-organization and physicochemical properties. Russian Chemical Bulletin. 2014;63(6):1399-1408.

23. Konovalov AI, Ryzhkina IS. Highly diluted aqueous solutions: Formation of nano-sized molecular assemblies (nanoassociates). Geochemistry International. 2014;52(13):1207-1226.

24. Konovalov A, Ryzhkina I, Maltzeva E, et al. Nanoassociate formation in highly diluted water solutions of potassiumphenosan with and without permalloy shielding. Electromagn Biol Med. 2015;34(2):141-146.

25. Finkelnburg W. Structure of Matter. USA: Academic Press; 1964. p. $412-414$.

26. http:// www.i-sis.org.uk/water4.php

27. Elia V, Napoli E, Niccoli M. Physical-Chemical Study of Water in Contact with a hydrophilic polymer: Nafion. J Therm Anal Calorim. 2013;112(2):937-944.

28. Elia V, Ausanio G, Ninno AD, et al. Experimental evidence of stable aggregates of water at room temperature and normal pressure after iterative contact with Nafion polymer membrane. Water. 2013;5:16-26.

29. Capolupo A, Giudice ED, Elia V, et al. Self-similarity properties of nafionized and filtered water and deformed coherent states. Int $J$ Mod Phys B. 2014:28(3).

30. Elia V, Napoli E, Niccoli M. Calorimetric and Conductometric titrations of nanostructures of water molecules in Iteratively Filtered Water. JTAC. 2013;111(1):815-821.

31. Cattaneo TMP, Vero S, Napoli E, et al. Influence of Filtration Process on Aqueous Nanostructures by NIR Spectroscopy. J Chem Chem Eng. 2011;5:1046-1052.

32. Elia V, Ausanio G, Ninno AD, et al. Experimental Evidences of Stable Water Nanostructures At Standard Pressure And Temperature Obtained by Iterative Filtration. Water. 2014;5:121-130.

33. Zheng JM, Chin WC, Khijniak E, et al. Surfaces and interfacial water: evidence that hydrophilic surfaces have long-range impact. Adv Colloid Interface Sci. 2006;127(1):19-27.

34. Giudice ED, Tedeschi A, Vitiello G, et al. Coherent structures in liquid water close to hydrophilic surfaces. J Phys Conf Ser. 2013;442:1-2.

35. Cacace CM, Elia L, Elia, V, et al. Conductometric and pHmetric Titrations of Extremely Diluted Solutions Using $\mathrm{HCl}$ Solutions as Titrant: A Molecular Model. J Mol Liq. 2009;146(3):122-126.

36. Elia V, Napoli E, Niccoli M. A molecular model of interaction between extremely diluted solutions and $\mathrm{NaOH}$ solutions used as titrant: Conductometric and pHmetric titrations. J Mol Liq. 2009; 148:45-50.

37. Elia V, Napoli E, Germano R. The 'Memory of water': an almost deciphered enigma - Dissipative structures in the extremely diluted aqueous solutions. Homeopathy. 2007;96(3):163-169.

38. Elia V, Napoli. Dissipative Structures in Extremely Diluted Solutions of Homeopathic Medicines: A Molecular Model based on PhysicoChemical and Gravimetric evidences. Int J Des Nat. 2010;5(19):39-48.

39. Elia V, Ausanio G, Gentile F, et al. Experimental evidence of Stable Water Nanostructures in Extremely Diluted Solutions at Standard Pressure and Temperature. Homeopathy. 2014;103(1):44-50. 
40. Yinnon TA, Elia V. Dynamics in perturbed very Dilute Aqueous Solutions: Theory and Experimental Evidence. Int J Modern Phys B $2013 ; 27: 1-35$.

41. Roy Rustum, Tiller WA, Bell Iris, et al. The structure of liquid water; novel insights from materials research; potential relevance to homeopathy. Materials Research Innovations. 2015:577-608.

42. Rey L. Thermo-luminescence of ultra-high dilutions of lithium chloride and sodium chloride. Physica-A. 2003;323:67-74.

43. Elia V, Ausanio G, Gentile F, et al. Experimental evidence of stable water nanostructures in extremely dilute solutions, at standard pressure and temperature. Homeopathy. 2014;103(4):44-50.
44. Barnard GO. Microdose paradox-a new concept. J American Inst Homeopathy. 1965;58(7):205-212.

45. Anagnostatos GS, Vithoulkas G, Garzonis P, et al. A working hypothesis for homoeopathic microdiluted remedies. The Berlin Journal of Research in Homoeopathy. 1991;1(3):141-147.

46. Kohanski MA, Dwyer DJ, Hayete B, et al. A Common Mechanism of Cellular Death Induced by Bactericidal Antibiotics. Cell. 2007;130(5):797-810.

47. http://health.howstuffworks.com/medicine/medication/question881.htm 